\title{
Growth of Partial Sums of Divergent Series
}

\author{
By R. P. Boas, Jr.
}

\begin{abstract}
Let $\Sigma f(n)$ be a divergent series of decreasing positive terms, with partial sums $s_{n}$, where $f$ decreases sufficiently smoothly; let $\varphi(x)=\int_{1}^{x} f(t) d t$ and let $\psi$ be the inverse of $\varphi$. Let $n_{A}$ be the smallest integer $n$ such that $s_{n} \geqslant A$ but $s_{n-1}<A$ $(A=2,3, \ldots)$; let $\gamma=\lim \left\{\Sigma_{1}^{n} f(k)-\varphi(n)\right\}$ be the analog of Euler's constant; let $m=[\psi(A-\gamma)]$. Call $\omega$ a Comtet function for $\Sigma f(n)$ if $n_{A}=m$ when the fractional part of $\psi(A-\gamma)$ is less than $\omega(A)$ and $n_{A}=m+1$ when the fractional part of $\psi(A-\gamma)$ is greater than $\omega(A)$. It has been conjectured that $\omega(A)=1 / 2$ is a Comtet function for $\Sigma 1 / n$. It is shown that in general there is a Comtet function of the form
\end{abstract}

$$
\omega(A)=\frac{1}{2}+\frac{1}{24}\left\{\left|f^{\prime}(m)\right| / f(m)\right\}(1+o(1)) .
$$

For $\Sigma 1 / n$ there is a Comtet function of the form $1 / 2+1 /(24 m)-\left\{1 /\left(48 m^{2}\right)\right\}(1+o(1))$. Some numerical results are presented.

1. Introduction. If $\Sigma_{n=1}^{\infty} f(n)$ is a divergent series of positive terms that approach 0 , one can measure how fast it diverges by seeing how fast the partial sums $s_{n}$ increase. Numerical data for representative series are given in the appendix to [4] (p. 69), but some of them are rather inaccurate. The present note grew out of an attempt to recompute this table. The results are given in the table on p. 259; they correct some of the entries in [4] and give a few more. The entries less than $10^{6}$ were found by direct machine evaluation of the partial sums; most of these were checked, and the other entries were obtained, by using Theorem 2 below, which is a generalization of known results for the harmonic series [2], [3]. The entries for the harmonic series (no. 4 in the table) were originally calculated by Wrench and published in [2].

A classical theorem of Maclaurin and Cauchy (see [4, p. 45]) states that if $f$ is positive and decreases to 0 , then $s_{n}-\int_{1}^{n} f(t) d t$ approaches a limit. When $f(n)=$ $1 / n$, this limit is Euler's constant $\gamma$; I use the same notation in the general case. The table includes approximations to $\gamma$ for each series.

Notation. $f$ is a positive decreasing function with $f(\infty)=0$, such that, at least for $n=1,2,3$, $\left|f^{(n)}(x)\right|$ decreases for large $x$ and is $O\left(f(x) x^{-n}\right)$, and with $\Sigma f(n)$ divergent. We define $\varphi(x)=\int_{1}^{x} f(t) d t ; \psi(y)$ is the inverse of $y=\varphi(x)$; we assume that $\psi^{\prime \prime \prime}$ is eventually monotonic. Let $s_{n}=\Sigma_{k=1}^{n} f(k)$ and $\gamma=\lim _{n \rightarrow \infty}\left(s_{n}-\varphi(n)\right)$. When $A$ is a positive integer, $n_{A}$ denotes the smallest integer $n$ such that $s_{n} \geqslant A$ but $s_{n-1}$ $<A$. 
For functions $f$ satisfying these hypotheses, the existence of $\gamma$ suggests that $\psi(A-\gamma)$ ought to be a good estimate of $n_{A}$.

THEOREM 1. For sufficiently large $A$, the number $n_{A}$ is one of the two integers closest to $\psi(A-\gamma)$.

Theorem 1 (with "sufficiently large" meaning "at least 2 ") was proved for the harmonic series by Comtet [3] ; this seems to have been the first really precise result in this direction.

Because of Theorem $1, n_{A}$ is either $[\psi(A-\gamma)]$ or $[\psi(A-\gamma)]+1$. Let us introduce a function $\omega$ such that the first case occurs when the fractional part of $\psi(A-\gamma)$ is less than $\omega(A)$; the second, when the fractional part of $\psi(A-\gamma)$ is greater than $\omega(A)$. Of course, $\omega$ is not uniquely determined. I propose to call such a function a Comtet function for $f($ or for $\Sigma f(n)$ ).

It has been conjectured that $\omega(A)=1 / 2$ is a Comtet function for the harmonic series, and proved [2] that this series has a Comtet function of the form $\omega(A)=1 / 2+$ $O\left(e^{-A}\right)$.

THEOREM 2. Every series of the form $\Sigma f(n)$ (with the hypotheses stated above) has a Comtet function of the form

$$
\omega(A)=\frac{1}{2}+\frac{1}{24}\left(\left|f^{\prime}(m)\right| / f(m)\right)(1+o(1)),
$$

where $m=[\psi(A-\gamma)]$.

For any specific $f$ we can improve Theorem 2 by more detailed calculation. We shall do this for the harmonic series.

THEOREM 3. For $\Sigma 1 / n$ there is a Comtet function of the form $1 / 2+1 /(24 m)-$ $\left(1 /\left(48 m^{2}\right)\right)(1+o(1))$. For $A \geqslant 2$ there is a Comtet function between $1 / 2+1 /(24 m)$ $-1 /\left(49 m^{2}\right)$ and $1 / 2+1 /(24 m)-1 /\left(47 m^{2}\right)$.

For larger values of $A$ the coefficients of $m^{-2}$ can be taken much closer together.

Theorem 3 does not disprove the conjecture that $\omega(A)=1 / 2$ is a Comtet function for the harmonic series, but it does seem to make it less plausible. It is conceivable that the fractional part of $e^{A-\gamma}$ never falls between $1 / 2$ and $1 / 2+1 /(24 m)-1 /\left(48 m^{2}\right)$. A machine computation for $A=20$ (1)200 found no exceptions; in fact, the cruder Comtet function found in [2] was more than adequate to determine $n_{A}$ for $A \leqslant 200$. The values of $n_{A}$ for $A=1(1) 20$ are given in [2] and reproduced in [9], sequence 1385; $n_{21}$ and $n_{22}$, calculated by H. P. Robinson, are given in a supplement to [9] . After the present paper had been submitted for publication, Robert Spira communicated to me the results of his computations in which he obtained $n_{A}$ for $A=100(100) 1000$, and also showed that there are no exceptions to the conjecture for $A \leqslant 1000$. Since $1 /(24 m)$ is about $2 \times 10^{-436}$ at this point, any exception to the conjecture will have the fractional part of $e^{A-\gamma}$ closer to $1 / 2$ than this, so that it seems unlikely that the conjecture will be disproved by computation.

For the series $\Sigma n^{-1 / 2}$, the corresponding conjecture is that $n_{A}$ is the closest integer to $(A-\gamma+2)^{2} / 4$, where now $\gamma=0.53964549119=2+\zeta(1 / 2)$ (as pointed out to me by John W. Wrench, Jr., who also provided me with the decimal approximation). I found no exceptions for $A=2(1) 1000$. 


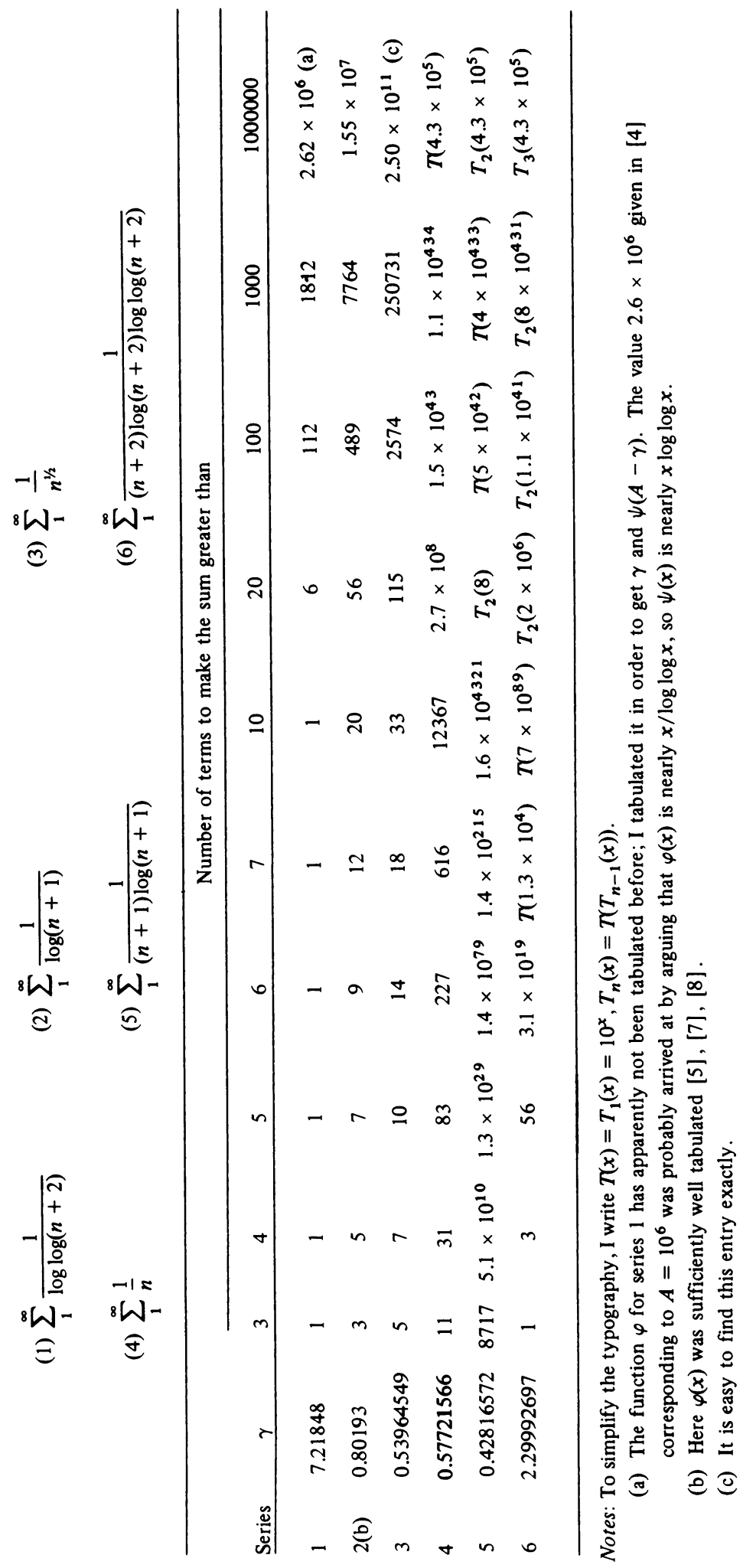


I am indebted to Dr. Wrench for the 150D value of $e^{-\gamma}$ which made the computations for the harmonic series possible. I am also indebted to Lester M. Carlyle, Jr., for communicating the results of his calculations which suggested the possibility of a result like Theorem 3.

I take this opportunity to note the following errata to [2]: In Theorem 1, last line, read $m$ for $n$ (twice). On p. 866, in the line before formula (1), read $-\frac{1}{8} n^{-2}$. On p. 868, lines 9 and 10 (statements (ii) and (iii)) read $m$ for $n$. On p. 865, first line, read "for $A=5,10,100$ his values are somewhat inaccurate."

2. Proof of Theorems 1 and 2. By the Euler-Maclaurin formula we can write

$$
s_{n}=\gamma+\varphi(n)+\frac{1}{2} f(n)+\frac{1}{12} f^{\prime}(n)+R_{n},
$$

where

$$
R_{n}=-\int_{n}^{\infty} f^{\prime \prime \prime}(t) P_{3}(t) d t
$$

and $P_{3}$ is the function of period 1 that is equal on $(0,1)$ to the Bernoulli polynomial $B_{3}(x) / 6$. (Notation for the $B$ 's as in [6] or [1].) We can estimate $R_{n}$ as in [6,pp. $538-539]$; it turns out that

$$
0<R_{n}<\frac{1}{720}\left|f^{\prime \prime \prime}(n)\right|=O\left(f(n) / n^{3}\right) .
$$

Suppose now that $n$ is any integer such that $s_{n} \geqslant A$. Put $\delta_{n}=1 / 2 f(n)+$ $f^{\prime}(n) / 12+R_{n}$; then from (2.1) we have $\varphi(n)+\delta_{n}>A-\gamma$, whence

$$
\psi\left\{\varphi(n)+\delta_{n}\right\}>\psi(A-\gamma) .
$$

We have $\varphi(n) \rightarrow \infty$ and $\delta_{n} \rightarrow 0$, so that it is reasonable to expand the left-hand side of (2.3) in a Taylor series with remainder of order 3 ,

$$
\psi\left\{\varphi(n)+\delta_{n}\right\}=\psi(\varphi(n))+\delta_{n} \psi^{\prime}(\varphi(n))+1 / 2 \delta_{n}^{2} \psi^{\prime \prime}(\varphi(n))+E_{n},
$$

where we may assume that

$$
\left|E_{n}\right| \leqslant \frac{1}{6} \delta_{n}^{3} \max \left\{\left|\psi^{\prime \prime \prime}(\varphi(n))\right|,\left|\psi^{\prime \prime \prime}(\varphi(n+1))\right|\right\},
$$

when $n$ is large enough (since we assumed that $\left|\psi^{\prime \prime \prime}\right|$ is monotonic). But $\psi(\varphi(n))=n$, $\psi^{\prime}(\varphi(n))=1 / f(n), \psi^{\prime \prime}(\varphi(n))=-f^{\prime}(n) / f(n)^{3}=O\left(n^{-1} f(n)^{-2}\right)$, and

$$
\psi^{\prime \prime \prime}(\varphi(n))=\left\{3 f^{\prime}(n)^{2}-f(n) f^{\prime \prime}(n)\right\} / f(n)^{5}=O\left(n^{-2} f(n)^{-3}\right)
$$

(and similarly for $\psi^{\prime \prime \prime}(\varphi(n+1))$ ). Hence, (2.4) becomes

$$
\psi\left\{\varphi(n)+\delta_{n}\right\}=n+\delta_{n} / f(n)-1 / 2 \delta_{n}^{2} f^{\prime}(n) / f(n)^{3}+E_{n},
$$

where $E_{n}=O\left(n^{-2}\right)$.

Now write $\delta_{n}=1 / 2 f(n)+f^{\prime}(n) / 12+R_{n}$ and multiply out $\delta_{n}^{2}$ in (2.6). We get

$$
\psi\left\{\varphi(n)+\delta_{n}\right\}=n+\frac{1}{2}-\frac{1}{24} f^{\prime}(n) / f(n)+O\left(n^{-2}\right),
$$


where the $O$-term can be calculated more precisely in any particular case. Thus, if $n$ is large enough, we can combine (2.3) and (2.7) to get

$$
n+\frac{1}{2}-\frac{1}{24} f^{\prime}(n) / f(n)+O\left(n^{-2}\right)>\psi(A-\gamma), \quad n>\psi(A-\gamma)-\frac{1}{2}+O\left(n^{-1}\right) .
$$

Consequently, if $m=[\psi(A-\gamma)]$ and $A$ is large enough, we have $n>m-1 / 2$. Since $n$ is an integer, this means that $n \geqslant m$. Now it was assumed that $s_{n} \geqslant A$; in particular, $n$ can be $n_{A}$, the smallest such index, and we conclude that $n_{A} \geqslant m$.

Similarly, if $n=n_{A}-1$, we have $s_{n}<A$, and so

$$
n_{A}-1<\psi(A-\gamma)-\frac{1}{2}+O\left(n^{-1}\right), \quad n_{A}<m+\frac{3}{2}+O\left(n^{-1}\right),
$$

whence $n_{A} \leqslant m+1$.

Consequently, we have shown that $m=[\psi(A-\gamma)] \leqslant n_{A} \leqslant m+1$ for large $A$, and this is the conclusion of Theorem 1 .

To go further, suppose that

$$
\psi(A-\gamma)>m+\frac{1}{2}+\left(\frac{1}{24}+\epsilon\right)\left|f^{\prime}(m)\right| / f(m), \quad \epsilon>0 .
$$

By definition, $s_{n} \geqslant A$ for $n=n_{A}$ and hence by (2.7), (2.3) and (2.8)

$$
n_{A}+\frac{1}{2}+\frac{1}{24}\left|f^{\prime}\left(n_{A}\right)\right| / f\left(n_{A}\right)+O\left(n_{A}^{-2}\right)>m+\frac{1}{2}+\left(\frac{1}{24}+\epsilon\right)\left|f^{\prime}(m)\right| / f(m) .
$$

Thus,

$$
n_{A}>m+\frac{1}{24}\left\{\frac{\left|f^{\prime}(m)\right|}{f(m)}-\frac{\left|f^{\prime}\left(n_{A}\right)\right|}{f\left(n_{A}\right)}\right\}+\epsilon \frac{\left|f^{\prime}(m)\right|}{f(m)}+O\left(n_{A}^{-2}\right) .
$$

We know that $m+1 \geqslant n_{A} \geqslant m$; since $\left|f^{\prime}(x)\right| / f(x)$ decreases, the expression in braces is nonnegative and so $n_{A}>m$ if $A$ is large enough, and (2.8) holds.

Similarly, if $s_{n}<A$ (as it is when $n=n_{A}-1$ ), we have

$$
n+\frac{1}{2}+\frac{1}{24} \frac{\left|f^{\prime}(n)\right|}{f(n)}+O\left(n^{-2}\right)<\psi(A-\gamma) .
$$

Supposing that

$$
\psi(A-\gamma)<m+\frac{1}{2}+\left(\frac{1}{24}-\epsilon\right)\left|f^{\prime}(m)\right| / f(m), \quad \epsilon>0
$$

we get

$$
n<m+\frac{1}{24}\left\{\frac{\left|f^{\prime}(m)\right|}{f(m)}-\frac{\left|f^{\prime}(n)\right|}{f(n)}\right\}-\epsilon \frac{\left|f^{\prime}(m)\right|}{f(m)}+O\left(m^{-2}\right) .
$$

Here $n=n_{A}-1<m$, so the expression in braces is not positive and consequently $n<m$, i.e., $n_{A}<m+1$. Therefore, $n_{A}=m$ under (2.10) if $A$ is large enough.

3. Proof of Theorem 3. We have $\varphi(x)=\log x, \psi(x)=e^{x}, \gamma=$ $0.5772156649 \ldots$. Then (2.1) becomes

$$
s_{n}=\gamma+\log n+\frac{1}{2 n}-\frac{1}{12 n^{2}}+R_{n}
$$

where 


$$
R_{n}=6 \int_{n}^{\infty} t^{-4} P_{3}(t) d t
$$

and by (2.2)

$$
0<R_{n}<\frac{1}{120} n^{-4}
$$

We now proceed as in Theorem 1 but take one more term in the Taylor series for $\psi(x)=e^{x}$. Here $\delta_{n}=(2 n)^{-1}-\left(12 n^{2}\right)^{-2}+R_{n}$ and

$$
\psi\left\{\varphi(n)+\delta_{n}\right\}=n e^{\delta} n=n\left(1+\delta_{n}+\frac{1}{2} \delta_{n}^{2}+\frac{1}{6} \delta_{n}^{3}+\epsilon_{n} n^{-4}\right),
$$

where

$$
0<\epsilon_{n} \leqslant \frac{1}{24} e^{\delta_{n}} \delta_{n}^{4}<\frac{1}{384} e^{1 /(2 n)}<0.0034
$$

if $n \geqslant 2$. Expanding the powers of $\delta_{n}$, we get

$$
\begin{aligned}
\psi\left\{\varphi(n)+\delta_{n}\right\}=n\{1 & +\frac{1}{2} n^{-1}-\frac{1}{12} n^{-2}+R_{n} \\
& +\frac{1}{2}\left(\frac{1}{4} n^{-2}+\frac{1}{144} n^{-4}+R_{n}^{2}-\frac{1}{12} n^{-3}+n^{-1} R_{n}-\frac{1}{6} n^{-2} R_{n}\right) \\
& +\frac{1}{6}\left[\frac{1}{8} n^{-3}+\frac{3}{4} n^{-2}\left(R_{n}-\frac{1}{12} n^{-2}\right)+\frac{3}{2} n^{-1}\left(R_{n}-\frac{1}{12} n^{-2}\right)^{2}\right. \\
& \left.\left.\quad+\left(R_{n}-\frac{1}{12} n^{-2}\right)^{3}+\epsilon_{n} n^{-4}\right]\right\} \\
=n+ & \frac{1}{2}+\frac{1}{24} n^{-1}-\frac{1}{48} n^{-2}+E_{n},
\end{aligned}
$$

where

$$
\begin{aligned}
n^{3} E_{n}= & \frac{1}{6} \epsilon_{n}-\frac{1}{144}+\frac{1}{576} n^{-1}-\frac{1}{10368} n^{-2} \\
& +R_{n}\left(n+\frac{1}{2}+\frac{1}{24} n^{-1}-\frac{1}{24} n^{-2}+\frac{1}{288} n^{-3}\right) \\
& +R_{n}^{2}\left(\frac{1}{2} n+\frac{1}{4}-\frac{1}{24} n^{-1}+\frac{1}{6} n R_{n}\right) .
\end{aligned}
$$

Since each of the expressions in parentheses is positive for $n \geqslant 2$, we get an upper bound for $n^{3} E_{n}$ by replacing $\epsilon_{n}$ and $R_{n}$ by their upper bounds from (3.1) and (3.2). The result is a decreasing function of $n$, so it is largest at $n=2$ and we get, after some calculation, $n^{3} E_{n}<0.005$. To get a lower bound for $n^{3} E_{n}$ we have only to replace $R_{n}$ and $\epsilon_{n}$ by 0 , and then we get

$$
n^{3} E_{n}>-\frac{1}{144}-\frac{1}{41472}>-0.007 .
$$

Using the upper bound, we obtain, for $n=n_{A}$,

$$
n+\frac{1}{2}+\frac{1}{24} n^{-1}-\frac{1}{48} n^{-2}+0.005 n^{-3}>e^{A-\gamma}
$$

Consequently, with $m=\left[e^{A-\gamma}\right]$, if 


$$
e^{A-\gamma}>m+\frac{1}{2}+\frac{1}{24} m^{-1}-\frac{1}{49} m^{-2}
$$

we have

$$
n>m+\frac{1}{24}\left(m^{-1}-n^{-1}\right)+\frac{1}{48}\left(n^{-2}-m^{-2}\right)+\left(\frac{1}{48}-\frac{1}{49}\right) m^{-2}-0.005 n^{-3}
$$

But we know that $n \geqslant m$; if we had $n=m$, (3.4) would yield

$$
0>\left(\frac{1}{48}-\frac{1}{49}\right) m^{-2}-0.005 m^{-3} \text {. }
$$

Now suppose that $A \geqslant 4$; then $m=\left[e^{A-\gamma}\right] \geqslant 30$, and so we would have

$$
0>\left(\frac{1}{48}-\frac{1}{49}\right)-(0.005) / 30>0.000425-0.00016 \text {. }
$$

This contradiction shows that $n>m$, so that $n=n_{A}=m+1$ under (3.3).

On the other hand, with $n=n_{A}-1$ we have

$$
n+\frac{1}{2}+\frac{1}{24} n^{-1}-\frac{1}{48} n^{-2}-0.007 n^{-3}<e^{A-\gamma} .
$$

If $n<m$, we have $n_{A}<m+1$ and so $n_{A}=m$, so we have only to exclude the possibility that $n=m$. If we suppose that $n=m$ and

$$
e^{A-\gamma}<m+\frac{1}{2}+\frac{1}{24} m^{-1}-\frac{1}{47} m^{-2},
$$

we then have

$$
m+\frac{1}{2}+\frac{1}{24} m^{-1}-\frac{1}{48} m^{-2}-0.007 m^{-3}<m+\frac{1}{2}+\frac{1}{24} m^{-1}-\frac{1}{47} m^{-2},
$$

that is, $1 / 47-1 / 48<0.007 m^{-1}$. If $A \geqslant 4$, we again have $m \geqslant 30$, and the last inequality says that $0.00043<0.00024$. Thus, the assumption that $n_{A}=m+1$ leads to a contradiction if (3.5) holds.

This establishes the second part of the theorem for $A \geqslant 4$; but it also holds, by direct computation, for $A=2,3$.

If we replace $1 / 47$ and $1 / 49$ by $1 / 48 \pm \epsilon$, we can take $\epsilon$ as small as we like if we take $A \geqslant A_{0}$, sufficiently large, and the first part of Theorem 3 follows.

Department of Mathematics

Northwestern University

Evanston, Illinois 60201

1. M. ABRAMOWITZ \& I. A. STEGUN (Editors), Handbook of Mathematical Functions With Formulas, Graphs and Mathematical Tables, Nat. Bur. Standards Appl. Math. Ser., 55, U. S. Government Printing Office, Washington, D.C., 1964. MR 29 \#4914.

2. R. P. BOAS, JR. \& J. W. WRENCH, JR., "Partial sums of the harmonic series," Amer. Math. Monthly, v. 78, 1971 , pp. 864-870. MR 44 \#7179.

3. L. COMTET, "Problem 5346," Amer. Math. Monthly, v. 74, 1967, p. 209.

4. G. H. HARDY, Orders of Infinitv. 2nd ed., Cambridge Univ. Press, New York, 1924.

5. K. A. KARPOV \& S. N. RAZUMOVSKIİ, Tablicy Integral'nogo Logarifma, Izdat. Akad. Nauk SSSR, Moscow, 1956, 319 pp. MR 19, 67; erratum, ibid., p. 1431. 
6. K. KNOPP, Theory and Application of Infinite Series, Blackie, London and Glasgow, 1928.

7. J. MILLER \& R. P. HURST, "Simplified calculation of the exponential integral," $M T A C$, v. 12, 1958, pp. 187-193. MR 21 \#3103.

8. Tables of Sine, Cosine and Exponential Integrals, Vols. I, II, National Bureau of Standards, WPA Project Nos. 765-97-3-10, 65-2-97-33, U. S. Government Printing Office, Washington, D. C., 1940. MR 2, 239, 366.

9. N. J. A. SLOANE, A Handbook of Integer Sequences, Academic Press, New York and London, 1973. MR 50 \#9760. 